\title{
The Influence of Magnetic Field on Pulse Distribution of Laser Diode
}

\author{
Samer H. Zyoud, Atef Abdelkader* \\ Department of Mathematics and Sciences, College of Humanities and Sciences, Ajman University, UAE
}

Received November 20, 2019; Revised December 11, 2019; Accepted December 28, 2019

Copyright $\bigcirc 2019$ by authors, all rights reserved. Authors agree that this article remains permanently open access under the terms of the Creative Commons Attribution License 4.0 International License

\begin{abstract}
The characteristics of a semiconductor laser diode (LD) are highly dependent on the magnetic field that applied on it. This study is valuable in several fields, including the existence of a magnetic field especially in a narrow region, which is difficult to detect by traditional techniques. This will lead to manufacture a micro-sensor to measure a weak magnetic field. In this study, we have investigated the influence of magnetic field, with an amplitude of (zero - $0.55 \mathrm{~T}$ ) on dynamical characteristics of semiconductor laser diode. This magnetic field has applied parallel and perpendicular on the axis of the active region of the laser diode (LD) chip. The LD used in this study is (Sanyo DL3149-056. ALGanP Index Guided Multiple Quantum will active laser and had threshold current of $11.15 \mathrm{~mA}$ and slope efficiency of $189.4 \mathrm{~mW} / \mathrm{A}$ ). It has been found that the full width half-maximum (FWHN), and the peak channel number (PCN) shift, were all increasing with the increased of magnetic field. Finally, the results show that while the peak of the pulse (light intensity) reduces with the increasing of magnetic field, the appearance of more than one peak (abnormalities) on the laser pulse has been founded clearly.
\end{abstract}

Keywords Magnetic Field, Semiconductor Laser, Active Layer, Threshold Current, Full Width Halfmaximum, Peak Channel Number Shift

\section{Introduction}

The threshold current density is one of the most important factors in the laser diode operating mechanism. Radiation is emitted from LD only when the current reaches the critical value due to absorption processes during the generation pairs (electron-hole) prevailing in the emission processes $[1,2]$. When the current reaches the threshold value, the gain is equal to the loss and the activated emissions are prevalent. When there is a slight increase in current after the threshold current, the slope efficiency (SE) increases and the spectral width of the emitted radiation increases [3].

The threshold current, Current density and slope efficiency are influenced by temperature [4], injection current [5], magnetic field [6], pressure [7], and thickness of the active layer [8]. The reason for this is loss of absorption, non-radioactive union processes, leakage of current and energy saturation.

The LD can be used as an optical sensor by connecting an optical fiber with a laser diode. LD sensitivity is detected by observing basic laser properties such as current density, barrier voltage or light intensity and observing changes in these properties due to the effect of magnetic field [9]. The laser beam may passes through a sample, and received at a detector connected to the spectral analyzer to study the change in pulse distribution, instability, and LD polarization rotation sensitivity when it passing through Faraday crystal by applying a magnetic field [10].

The effect of magnetic field at the level of Fermi [11] is performed at a value near the barrier's voltage. The magnetic field has a distinct effect on the propagation of carriers across the active layer. This process is evident near the potential voltage of the barrier in the LD active propagation region [12]. The magnetic field affects the current density of the LD, and therefore affects the temperature near the active layer. As a result, while the output power decreases by the rise of magnetic field, the threshold current of the LD increased [13]. When a magnetic field is exposed to LD chip, it causes a change in the threshold current, output power, barrier voltage, and current density caused by Lorentz force that creating a non-homogeneous region in the refractive index, gain and loss, and temperature inside and outside the active layer [14]. 


\section{Experimental Setup}

In our previous paper [13], we have prepared a chip of LD. The LD used has a wavelength of $670 \mathrm{~nm}$ and emission power of $1.00 \mathrm{~mW}$ above the threshold current as shown in Fig. 1

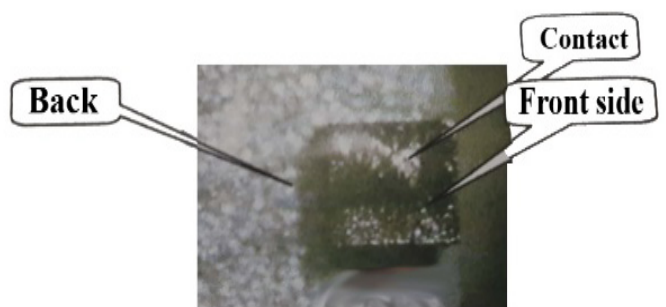

Figure 1. Pictures of the chip of LD before and after functioning.

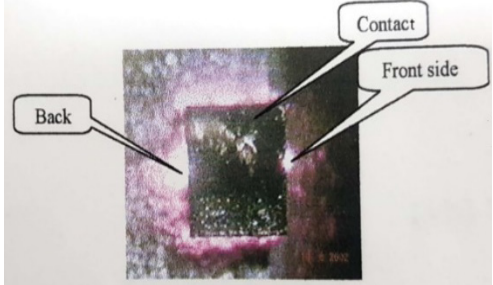

Front side (output laser beam), back side (area of unwanted reflections) and the electric contact (electrons injection)

To study the resulting changes in the pulse distribution of the LD under the influence of the magnetic field, an electronic circuit and a thermal base were designed to control the stability of laser's injection current and temperature, respectively as shown in Fig. 2.

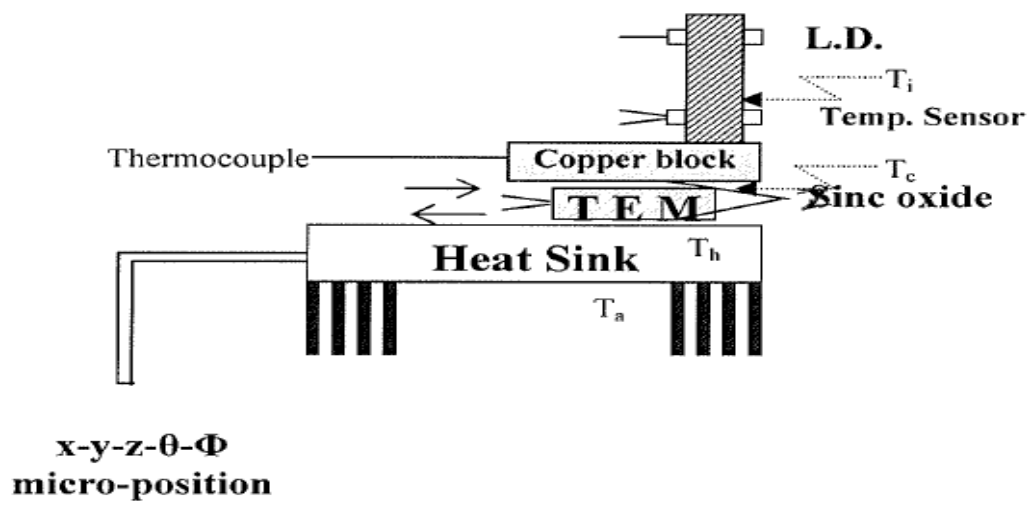

Figure 2. A thermal base constructed consisting of a LD base [13]

The laser pulse was received by an electronic silicon detector (RS-UKBPX 65 and has a wavelength $850 \mathrm{~nm}$, the climb time $0.5 \mathrm{~ns}$, voltage $5.0 \mathrm{~V}$, and response power $0.55 \mathrm{~A} / \mathrm{W}$ ), through an optical fiber that was prepared as shown in Fig 3.

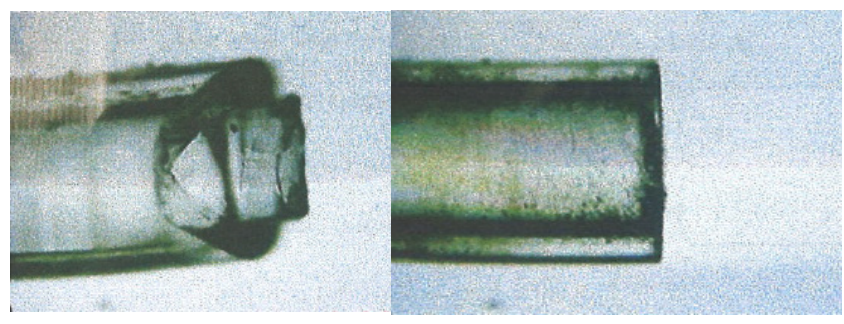

Figure 3. The Optical fiber before and after cutting and polishing process [13].

A magnetic field generator (Electromagnet LH/51450) of a maximum current operating 12 A was used to give a magnetic field $(0.87 \mathrm{~T})$. Once the distance between the poles of the magnetic field generator is $7.5 \mathrm{~mm}$, a Tesla-ammeter PHYWE / 11749.93 with Probe PHYWE / 11749.01 used to measure the magnetic field strength and its stability over time.

In our previous paper, a linear relationship has been found between the magnetic field and the current passing through its coils, at different values of the distance (air gap) between the poles [13] while relation of magnetic field with the gap between the poles is exponential according to the relationship, $B=0.983 e^{-0.572 x}$ [13]. The plot of magnetic field and time at different values of the gap and constant current of $10 \mathrm{~A}$ has been also settled and shows the stability of the magnetic field with time. 
The base of LD mounted on high-stability ( $\mathrm{x}-\mathrm{y}-\mathrm{z}-\Theta-\varnothing)$ holder to enable the installation of a LD device between the poles of magnetic field generator with a gap of $1 \mathrm{~cm}$, to obtain a best alignment between the beam of LD and the optical fiber. The magnetic field generator gives $\mathrm{B}=0.55 \mathrm{~T}$ at operating current of $10 \mathrm{~A}$, and the air gap between the poles of magnetic field generator of $x=1 \mathrm{~cm}$. An optical fiber used to direct the beam of LD, where it is difficult to align the laser device installed between the polar magnetic field generator and the detector. Optical fiber ends are installed on 3D (x, y, z) highly stable to get the best alignment. The detector diode is also fixed on a $3 \mathrm{D}(\mathrm{x}, \mathrm{y}, \mathrm{z})$ stabilizer with high stability so that it can receive the maximum intensity of the laser light through the optical fiber.

The digital voltmeter (DM1) used to measure the injection current while DM2 to measure the operating voltage of LD and DM3 to measure the optical power received on the photoreceptor after calibration with voltages. The oscilloscope (CRO) used to monitor the pulse of the current. Multichannel Analyzer (MCA) used to identify pulse shape, as shown in fig. (4).

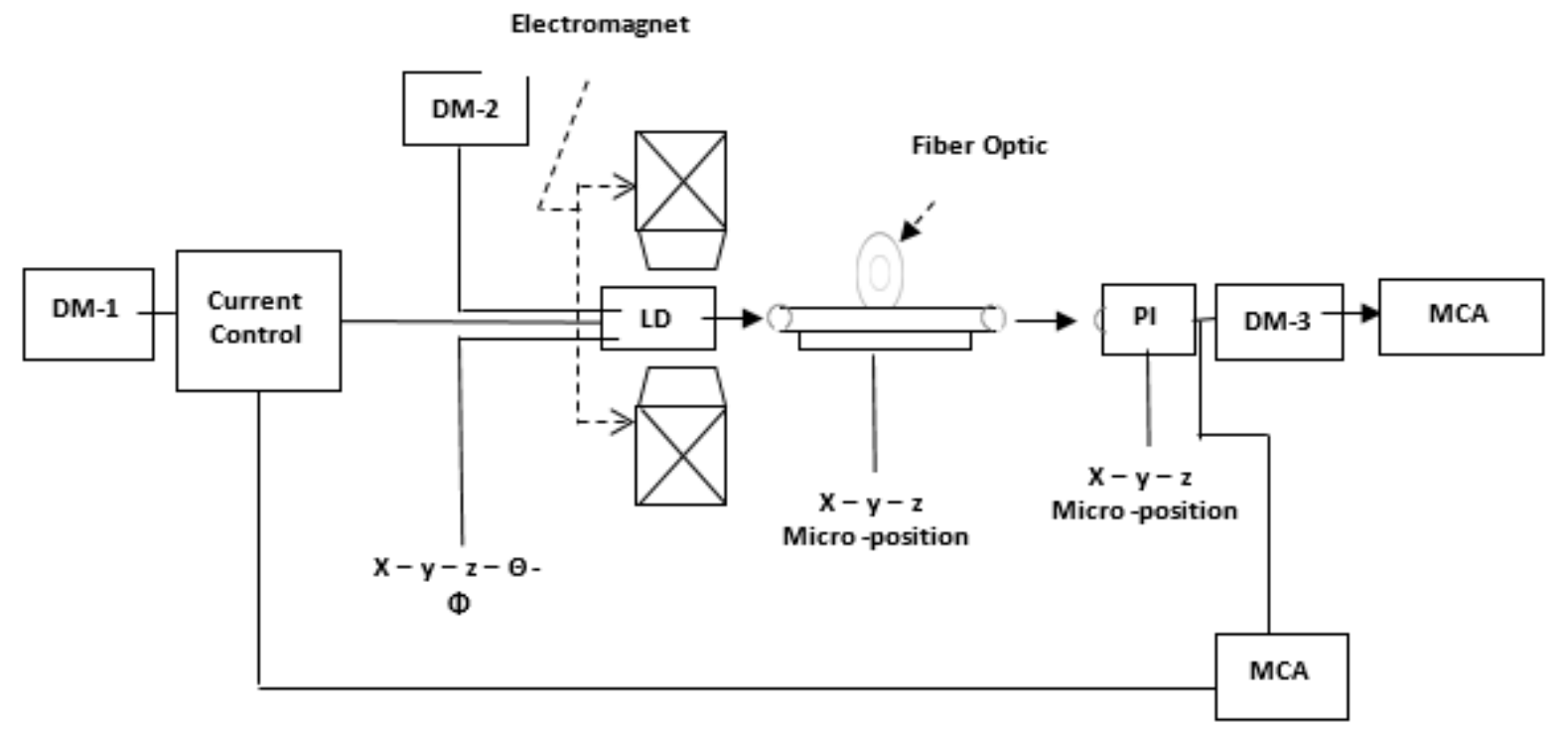

Figure 4. Setup of the used system to investigate the effect of the magnetic field on LD's pulse distribution.

\section{Results and Discussion}

Fig. 5 shows the distribution analysis of pulsed for beam of LD without any external effect at $\mathrm{T}=300 \mathrm{~K}$. The distribution shows a caustic and regular form at the threshold current $1.1 \mathrm{~A}$, while the highest pulse peak is located at channel No. $=49$, the frequency $1 \mathrm{MHz}$, the time width is $500 \mathrm{~ns}$ and the capacity is $2.5 \mathrm{~V}$. The pulse was received, and displayed by multi-channel analyzer (MCA).

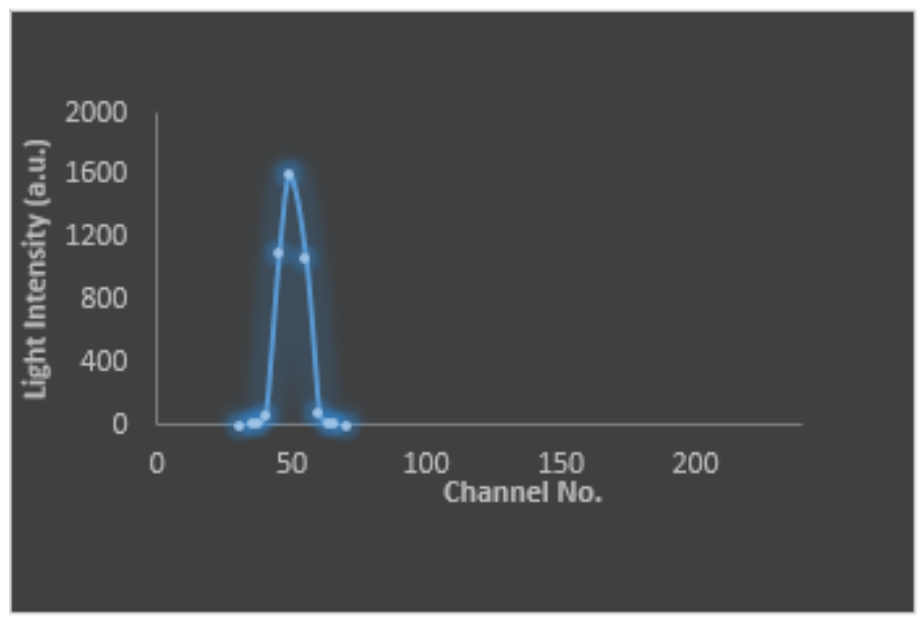

Figure 5. The distribution analysis of pulsed for beam of $\mathrm{LD}$ without any external effect. $(\mathrm{T}=300 \mathrm{~K})$ 
Figures (6a-c) and (7a-c) display the pulse distribution of the LD with different values of the magnet field applied perpendicular and parallel on the axis of the active region of LD chip. The results show significant changes in the form of pulse with the increases of magnetic field. These changes include increase in the Full Width Half Maximum (FWHN), shifting the peak channel number (PCN) towards the right, dropping in the top of the pulse and distortion of the pulse form by the appearance of number of peaks and dips of the pulse due to vibrations of the pulse. It is clearly that the changes in the form of pulse when magnetic field is projected perpendicular is more than when its projected parallel to the axis of the LD chip. This can be conveying to magnetic field influence; while the temperature is staying constant, the influence of the magnetic field on the mechanisms of loss in the laser gap affecting the current density of laser diode and thus affect the temperature near the active area [15].
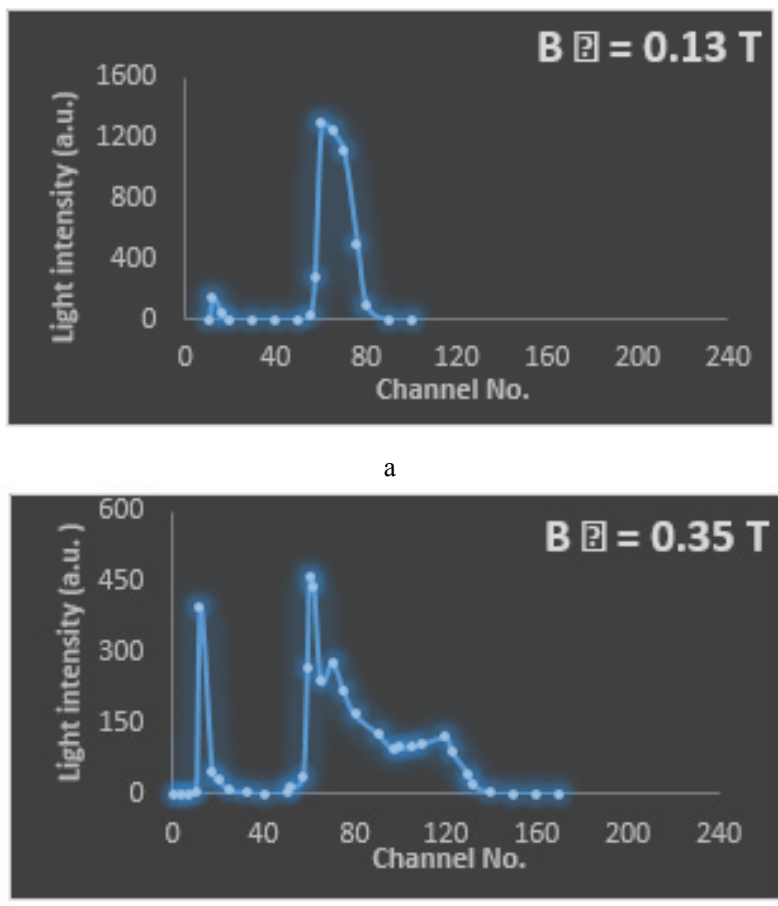

b

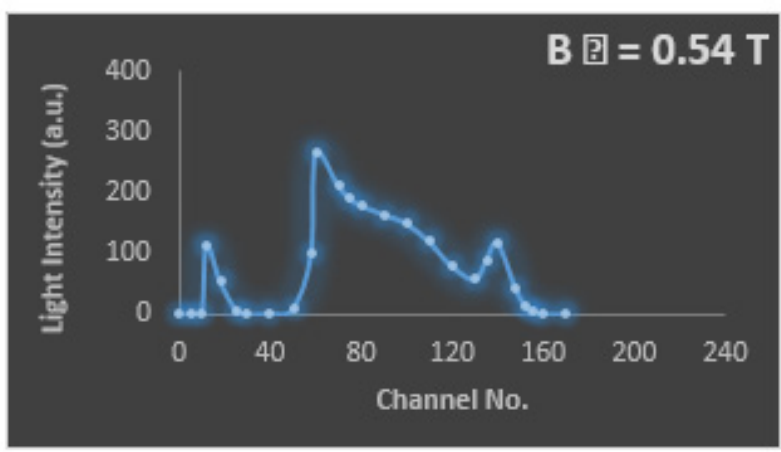

c

Figure 6. a, b, c: The pulse distribution of the LD at different values of magnet field. (B $\square$ on the axis of LD chip).

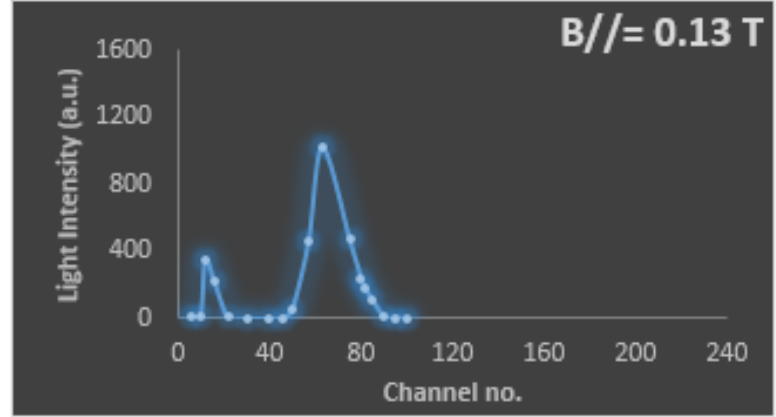

$\mathrm{a}$

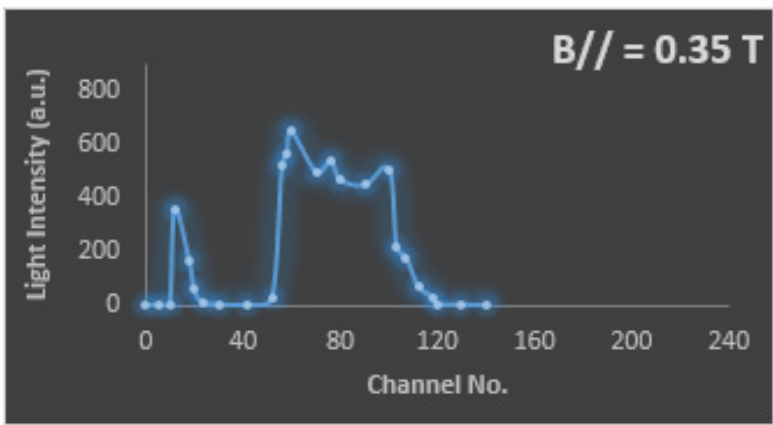

b

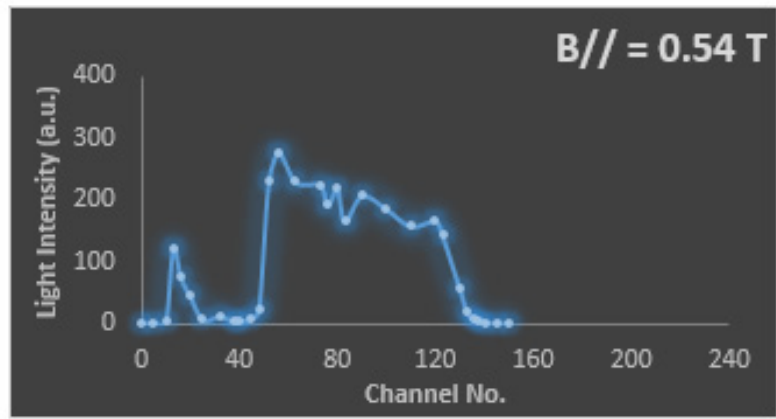

Figure 7. $a, b, c:$ : the pulse distribution of $L D$ at different values of magnet field. (B// on the axis of LD chip)

The relation between FWHN and the PCN shift of the LD with magnetic field was carefully investigated. Figure 8 and 9 show a linear relationship; any increase in magnetic field lead to increases in FWHN and PCN shift of LD. The reason for that is due the magnetic field, which leads to the suppressed of the propagation current, and therefore induced change in the current flow path that caused by Lorentz force producing an increase in active region's current density. Inhomogeneous region in the refractive index and the gain, loss and distribution of carriers inside and outside of the active region is created by the magnetic field and therefore a change occurs in the sites of peaks. 


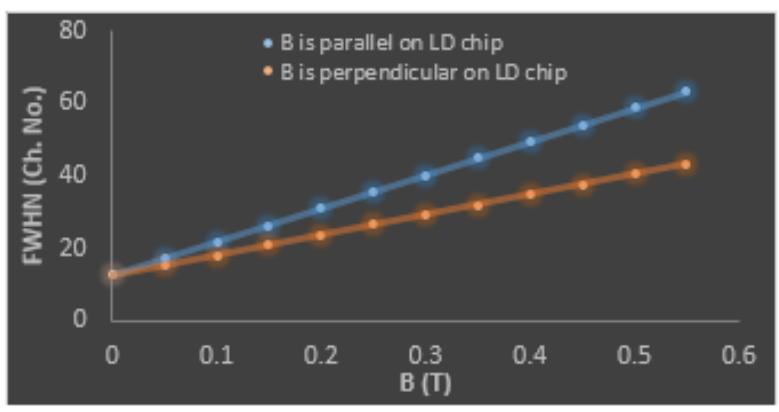

Figure 8. FWHN of the pulse of the LD against increasing of the magnetic field strength.

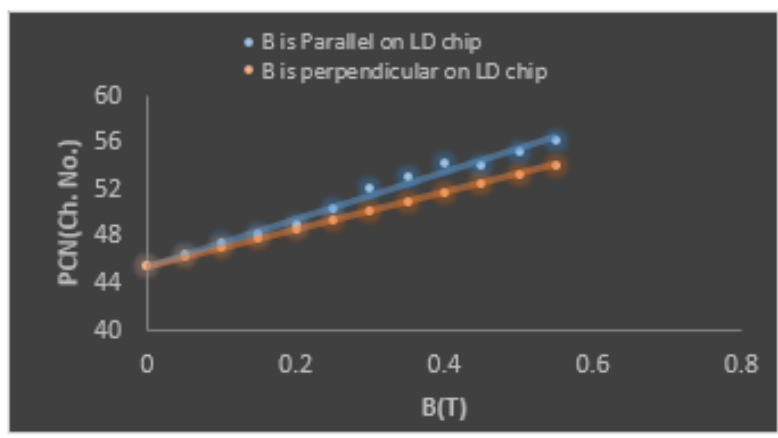

Figure 9. PCN of the LD against increasing of the magnetic field strength

Finally, figure 10 represent the light intensity against the increasing of magnetic field strength when the field projected perpendicular and parallel to the axis of the LD chip. The decreasing in light intensity can be attributing to the magnetic field effect, while temperature is remaining constant. This is due to the magnetic field influence on the mechanisms of loss in the laser gap that lead to changing in current density of LD and thus effect on the temperature near the active region.

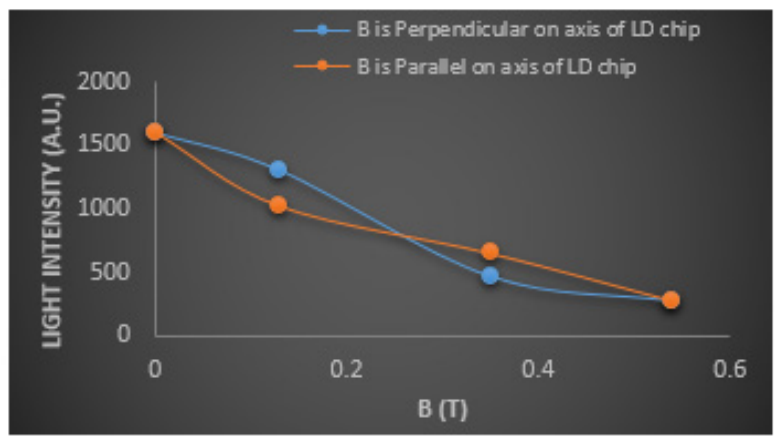

Figure 10. The light intensity of the LD against increasing of magnetic field strength.

\section{Conclusions}

The main characteristics of the semiconductor laser, the pulse distribution analysis of the semiconductor laser (Full
Width Half Maximum of the pulse of the LD and peak channel number shift), were studied when the magnetic field was projected (perpendicular and parallel) at the axis of the active region of the laser chip. Any practical measurements must be founded on an experimental system with special characteristics to preserve current and temperature without change in order to ensure that the influence on the laser properties is a magnetic field effect.

We found that, when the magnetic field that projected perpendicular on the axis of LD chip is increasing, FWHN of the pulse of LD increasing by $55.627 \mathrm{Ch}$. No. /T and $P C N$ shift increasing by $15.747 \mathrm{Ch}$. No. $/ T$. While when the magnetic field projected parallel to the axis of $\mathrm{LD}$, the $F W H N$ of the pulse of the LD increase by $92.292 \mathrm{Ch}$. No. /T. and the $P C N$ shift increasing by $13.534 \mathrm{Ch}$. No. /T. Finally, the results show that, any increasing in the magnetic field that projected perpendicular and parallel to axis of LD chip will lead to decreasing in the light intensity.

\section{REFERENCES}

[1] Cunyun, Y. (2004). Tunable external cavity diode lasers. World Scientific.

[2] Liu, A. Q., \& Zhang, X. M. (2006). A review of MEMS external-cavity tunable lasers. Journal of Micromechanics and Microengineering, 17(1), R1.

[3] Schultz, C. M. (2013). Analysis and mitigation of the factors limiting the efficiency of high power distributed feedback diode lasers (Doctoral dissertation, Technische Universität Berlin),

[4] Azawe, M. I. (2013). Temperature dependence of wall-plug efficiency of high power laser diodes. International Journal of Physical Sciences, 8(10), 362-370.

[5] [5]Al Roumy, J., Perchoux, J., Lim, Y. L., Taimre, T., Rakić, A. D., \& Bosch, T. (2015). Effect of injection current and temperature on signal strength in a laser diode optical feedback interferometer. Applied optics, 54(2), 312-318.

[6] Seto, Y., Miyamoto, T., Sato, T., Ohkawa, M., \& Maruyama, T. (2005, April). Magnetic field effects on semiconductor laser characteristics. In Physics and Simulation of Optoelectronic Devices XIII (Vol. 5722, pp. 90-98). International Society for Optics and Photonics.

[7] Hung, C. T., \& Lu, T. C. (2013). 830-nm AlGaAs-InGaAs graded index double barrier separate confinement heterostructures laser diodes with improved temperature and divergence characteristics. IEEE Journal of Quantum Electronics, 49(1), 127-132.

[8] Thahab, S. M. (2012). Characterization Parameters of (InGaN/InGaN) and (InGaN/GaN) Quantum Well Laser Diode. In Semiconductor Laser Diode Technology and Applications. IntechOpen.

[9] Matsumoto, S., Tamura, Y., \& Nishizawa, K. (2007, June). Laser diode and photodiode modules and analogue circuits training for the optical fiber transmission practice system. 
In Education and Training in Optics and Photonics (p. EMB4). Optical Society of America.

[10] Subkhangulov, R. R., Mikhaylovskiy, R. V., Zvezdin, A. K., Kruglyak, V. V., Rasing, T., \& Kimel, A. V. (2016). Terahertz modulation of the Faraday rotation by laser pulses via the optical Kerr effect. Nature Photonics, 10(2), 111.

[11] Kim, K. H., Yamaguchi, M., Arai, K. I., Nagura, H., \& Ohnuma, S. (2003). Effect of radio-frequency noise suppression on the coplanar transmission line using soft magnetic thin films. Journal of applied physics, 93(10), $8002-8004$

[12] Gsponer, A. (2004). Physics of high-intensity high-energy particle beam propagation in open air and outer-space plasmas. arXiv preprint physics/0409157.

[13] Samer H. Zyoud, Atef Abdelkader. (2019). Characteristics of semiconductors laser Under Influence of Magnetic field. Journal of Advenced Research in Dynamical \& Control Systems, Vol. 11, 10-Special Issue.

[14] Jooss, C., Albrecht, J., Kuhn, H., Leonhardt, S., \& Kronmüller, H. (2002). Magneto-optical studies of current distributions in high-Tc superconductors. Reports on progress in Physics, 65(5), 651.

[15] Fukuda, M., Mishima, T., Nakayama, N., \& Masuda, T. (2010). Temperature and current coefficients of lasing wavelength in tunable diode laser spectroscopy. Applied Physics B, 100(2), 377-382. 